\title{
Focus on neural control of feeding
}

\author{
We present a special focus that highlights research on the role of the CNS in the regulation of feeding behavior and \\ how disruption of such regulation can lead to obesity.
}

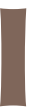
n 2005, Nature Neuroscience ran a focus issue on feeding regulation and obesity, commissioning Reviews and Perspectives that examined our understanding of neural regulation of feeding. Seven years later, we're revisiting the topic in this special focus. Since 2005 , the rate of obesity among adults and children has continued to climb both in the US and worldwide (http://www.cdc.gov/, http://www. who.int/). In 2005, only 3 of the 50 states in the US had obesity rates above $30 \%$, whereas that number had risen to 12 in 2011. Although this reflects a lack of progress in combating obesity in the last 7 years, our understanding of how the brain regulates feeding behavior and energy balance has advanced substantially over this period. In this issue, we present a collection of Reviews highlighting some of the recent developments in the field.

Although obesity and metabolic syndrome are often seen as diseases of the body, the CNS is important for regulating feeding behavior and metabolism. The arcuate nucleus of the hypothalamus is recognized as the brain's command center for controlling energy balance. Along with other hypothalamic and brainstem nuclei, the neurons of the arcuate nucleus coordinate energy intake and expenditure. To maintain homeostasis, neurons in these areas must integrate information from other regions of the brain, as well as hormonal and nutrient signals from the periphery. On page 1350, Kevin Williams and Joel Elmquist discuss the role of peripheral signals in regulating energy balance and how these signals are integrated centrally. These signals include the adipose tissue-derived hormone leptin, as well as ghrelin, insulin, peptide YY, cholecystokinin and others derived from the gastrointestinal system. Neurons in the hypothalamus and brainstem contain receptors for these factors, and research is beginning to elucidate the physiological effects that these peripheral factors have on different populations of neurons throughout the brain.

Beyond the immediate excitatory or inhibitory effects of feedingrelated hormones and neurotransmitters, these factors can also exert long-term effects on hypothalamic neurons. In their Review, Lori Zeltser, Randy Seeley and Matthias Tschöp discuss plasticity of the neuronal circuits regulating energy balance. In this relatively new field, research has shown that the same signals can have different effects depending on whether they are received under fed or fasted conditions. The mechanisms behind the effects of metabolic state on feeding circuits are still under intense investigation, but evidence has emerged that both short- and long-term changes in metabolic state can lead to synaptic rearrangements and changes in the excitability of neurons in the hypothalamic circuit.
Many of the neurotransmitters and hormones that regulate feeding behavior were originally identified through mouse genetic studies. Two spontaneous mutations in mice that resulted in severe obesity were traced to the genes encoding the hormone leptin and its receptor. Since then, modern mouse genetics has also allowed researchers to identify specific cell populations in which leptin and other signals are required to maintain energy homeostasis. On page 1343, Giles Yeo and Lora Heisler discuss what we have learned about the brain's regulation of appetite from genetic studies. In addition to highlighting the role of mouse genetics in dissecting which neurotransmitters and hormones act in distinct cell populations to regulate feeding behavior, they also discuss more recent attempts at using human genetics to identify new molecules that may be involved in maintaining energy balance. Although Mendelian forms of severe obesity in humans do exist, they are rare. Instead, recent genome-wide association studies have attempted to find genetic variations that contribute to common obesity. Several loci have been identified, although how these variations lead to obesity will have to be the focus of future study.

Despite the central role of the hypothalamus in energy balance, another circuit has recently received much attention. The comparison between the uncontrolled intake of food and drugs has led to a spotlight on reward circuitry and its contribution to the regulation of feeding behavior. Although parallels have been drawn between obesity and addiction in recent years, Ralph DiLeone, Jane Taylor, and Marina Picciotto argue that a more nuanced interpretation of the similarities and differences between these two diseases is warranted. In their Review, they discuss both similarities and differences in the responses to food and drugs of abuse, focusing on behavioral and circuit-level responses. Although the two fields of research may mutually benefit from comparisons of the two diseases, they urge caution in thinking of obesity as a food addiction.

Although our understanding of the brain regulation of feeding and energy balance has improved substantially in recent years, this has not yet translated into new treatments or therapies to mitigate obesity. As with many fields, further research seems to reveal ever more layers of complexity in the interaction between the brain and the body regarding the regulation of energy balance. Our hope is that these Reviews will inspire further research into the mechanisms underlying the central control of feeding and energy expenditure, which may lead to more promising treatments for obesity, and that they give our readers a glimpse of some of the exciting recent research in this field. 